\title{
Regressgefahr Arzneibezug
}

\section{Günstigste Quelle entscheidend}

\author{
Zur wirtschaftlichen Verordnungsweise gehört laut Urteil des \\ Sozialgerichts Magdeburg nicht nur die Wahl des richtigen \\ Arzneimittels, sondern auch die der preisgünstigsten Bezugsquelle. \\ Sonst droht ein Regress.
}

$\mathrm{D}$ ie Gefahren, die sich aus den Möglichkeiten des Direktbezugs bestimmter Arzneimittel beim Hersteller und aus Verträgen zwischen Krankenkassen und Apotheken über die rabattierte Abgabe bestimmter Arzneimittel, insbesondere von Impfstoffen, ergeben, sind in der Realität der Wirtschaftlichkeitsprüfung angekommen. Das Sozialgericht Magdeburg hat entschieden, dass zur wirtschaftlichen Verordnungsweise von Arzneimitteln auch die Wahl der preisgünstigsten Bezugsquelle gehört (Az.: S 21 KA 97/08). Nach diesem Urteil ist eine Arzneimittelverordnung auch dann unwirtschaftlich, wenn eine gleich geeignete, aber kostengünstigere Bezugsform zur Verfügung stand. Dies kann beim Bezug von Gerinnungsfaktorzubereitungen, Zytostatikazubereitungen und im Rahmen von Sprechstundenbedarfsverordnungen zukünftig eine erhebliche Rolle spielen.

Pharmazeutische Unternehmer dürfen bestimmte Arzneimittel direkt an Ärzte abgeben. Dies ist in Paragraf 47 Arzneimittelgesetz (AMG) geregelt und betrifft vor allem Gerinnungsfaktorenzubereitungen, radioaktive Arzneien und Impfstoffe. Bei dem Bezug von Zytostatikazubereitungen darf der Arzt unmittelbar mit einer herstellenden Apotheke zusammenarbeiten. In diesen Bereichen haben sich, auch durch die Ergebnisse von vergaberechtlichen Ausschreibungsverfahren der Kassen unterschiedliche Preise für identische Arzneimittel entwickelt.

\section{Auf die Infos von KV und Kassen achten}

So bezog die vor dem Sozialgericht Magdeburg klagende Ärztin ihre Gerinnungsfaktorprodukte über eine öffentliche Apo- theke. Sie war zuvor von Mitarbeitern einer Krankenkasse darüber informiert worden, dass der Direktbezug bei einem Hersteller wirtschaftlicher sei. Die Ärztin machte von der Möglichkeit des Direktbezuges aber keinen Gebrauch. Auf Antrag der Krankenkasse setzten die Prüfgremien einen Regress in Höhe der durch den Apothekenbezug entstandenen Mehrkosten fest. Das Sozialgericht bestätigte die Rechtmäßigkeit dieses Regresses.

Seit 2007 sind die Kassen und die Kassenärztlichen Vereinigungen (KVen) gemäß Paragraf 73 Abs. 8 SGB V verpflichtet, die Vertragsärzte auch über preisgünstige Bezugsquellen zu informieren. Jedenfalls seit Existenz dieser Informationspflicht wird man wohl nicht umhin können, die Auswahl der günstigsten Bezugsquelle als Bestandteil des Wirtschaftlichkeitsgebotes anzusehen. Dies gilt jedenfalls für die Fälle, in denen die eigene KV oder eine Krankenkasse den Vertragsarzt konkret über einen günstigen Bezugsweg informiert hat. Der Arzt wird demgegenüber nicht verpflichtet sein, sich aus nicht öffentlich zugänglichen Quellen, beispielsweise durch individuelle Anfragen bei einem Hersteller, über die jeweils aktuellen Preise zu informieren und diese zu vergleichen.

\section{Am besten gleich den günstigsten Weg wählen}

Allerdings machen die KVen und Krankenkassen bereits in vielen Fällen von ihrer Informationsmöglichkeit Gebrauch. Durch die Krankenkassen geschieht dies vor allem dann, wenn sie im Vergabeverfahren mit bestimmten Herstellern oder Apotheken Rabattverträge geschlossen haben.

Auch beim Bezug von Sprechstundenbedarf informieren die KVen häufig über

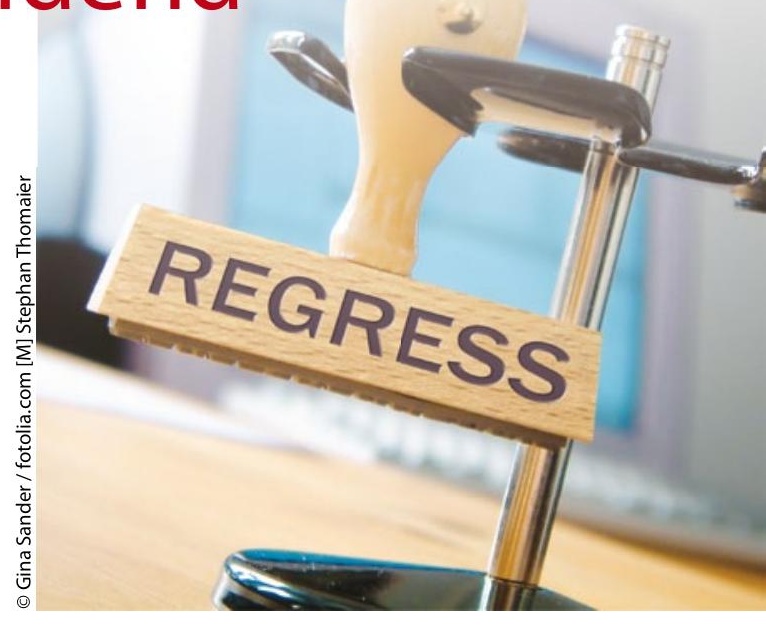

günstige Anbieter oder bieten diesbezüglich telefonische Beratungen an. Nach der ersten gerichtlichen Entscheidung zum Regress wegen der Wahl einer unwirtschaftlichen Bezugsquelle sollte jeder Vertragsarzt aus eigenem Interesse darauf achten, von den Informationsangeboten Gebrauch zu machen und keinesfalls gegen einen ausdrücklichen Hinweis einen anderen als den wirtschaftlichsten Bezugsweg zu wählen.

Da es nicht um den Wirkstoff, sondern nur um den Bezugsweg geht, können individuelle Umstände oder Praxisbesonderheiten nämlich nicht mit Aussicht auf Erfolg geltend gemacht werden. Man könnte aus der Tatsache, dass erst im Jahr 2012 ein Urteil eines Sozialgerichts zum Regress bei unwirtschaftlichem Bezugsweg eines Arzneimittels ergeht, die Schlussfolgerung ziehen, das Wirtschaftlichkeitsgebot werde bei der Auswahl der Bezugsquelle weitestgehend beachtet. Diese Schlussfolgerung könnte aber voreilig sein. Eventuell richtet sich der Fokus der Krankenkassen erst in jüngster Zeit auf dieses Detail des Wirtschaftlichkeitsgebotes. Es ist klüger, sogleich die günstigste Bezugsquelle zu wählen als später die Mehrkosten aus eigener Tasche zu zahlen.

\section{Dr. Ingo Pflugmacher}

Fachanwalt für Medizin- und Verwaltungsrecht

Partner der Anwaltskanzlei

Busse \& Miessen, Bonn 